\title{
MULTIPACTING SIMULATIONS FOR SUPERCONDUCTING CAVITIES AND RF COUPLER WAVEGUIDES*
}

\author{
R.L. Geng ${ }^{\dagger}$, LEPP, Cornell University, Ithaca, NY 14853, USA
}

\section{Abstract}

Multipacting (MP) has recently received a renewed interest in the community of superconducting RF. In this paper, I will overview the developments of MP simulations for SRF cavities and RF couplers, summarize the simulation results, compare experiment results with simulation predictions and examine the algorithms. After identifying the discrepancy between the predictions and experiments, suggestions are given to further improve the simulations.

\section{INTRODUCTION}

MP is a phenomenon of resonant secondary emission multiplication, first described by Farnsworth in 1934 [1]. The operating mechanism of MP is that participating electrons, driven by RF fields, impact a surface and release secondary electrons, which in turn driven by RF fields, are made to impact again and release more secondary electrons. This process will go on resonantly (electrons are "synchronized" to RF fields) until the number of electrons are saturated due to some limiting mechanism. MP may occur in an array of evacuated RF devices and may involve one surface or two surfaces.

MP was intensively studied in the 40s and 50s for a specific case, namely two-sided MP in a parallel plate, in the context of high frequency gaseous discharge. Gill and von Engels [2] and others developed theoretical formulations for the parallel plate MP. Hatch and Williams [3] extended the work and generalized the formulation to allow construction of MP susceptible zones, including higher order MP. These formulations have now become known as "constant k theory" to reflect the adoption of a constant ratio between the electron velocity upon impact $\left(v_{i}\right)$ to the velocity at emission $\left(v_{0}\right)$. Theoretical treatments were also developed by Krebs and Meerbach [4] and by Tamagawa [5]. Instead of imposing a constant ratio to $v_{i} / v_{0}$, they adopted a constant $v_{0}$, typically equivalent to a few eV. In the $80 \mathrm{~s}$, the "constant $v_{0}$ " theory was advanced by Shemelin [6] and by Vaughan [7] in which important effects like the phase stability and the MP saturation due to space charge are explicitly examined. In most MP simulation studies to be presented in this paper, a constant $v_{0}$ is adopted.

Simulation studies of MP in the superconducting RF (SRF) community can be traced back to the 70s, when MP was a major SRF cavity performance limitation [8]. With the aid of computer simulations, a new type of MP, onepoint MP, was discovered in pill-box like cavities by Lyneis

\footnotetext{
* Work supported by NSF

†rg58@cornell.edu
}

et al. [10] and in muffin-tin cavities by Padamsee et al. [11]. Klein and Proch showed through simulation studies that a spherical cavity shape is free of one-point MP [12]. This finding resulted a breakthrough in SRF cavity performance. MP then became less of a concern until 1984, when two-point MP was discovered in a $500 \mathrm{MHz}$ spherical cavity by Weingarten [14] through a combined experimental and simulation study. Owing to its nature, two-point MP did not become a limiting mechanism.

MP has recently received a renewed interest in the SRF community. This is mainly due to the fact that this mechanism is found to cause harmful RF breakdown in today's multi-hundred $\mathrm{kW}$ couplers for SRF cavities. In addition, two-sided MP has been identified in elliptical cavities for a broad frequency range from $200 \mathrm{MHz}$ to $3000 \mathrm{MHz}$. Numerical simulations have been advanced in the past decade, allowing systematic studies of MP characteristics of new RF structures [15]. Several simulation tools now exist to evaluate MP likely-hood and to predict MP suppression methods [16]. Important experimental verifications of the predictions made by these codes have been brought forward since last year. In this paper, I will overview the developments of MP simulations in SRF cavities and couplers, summarize the simulation results, compare experiment results with simulation predictions and examine the algorithms. Suggestions are given to improve the simulations.

\section{MP SIMULATIONS FOR SRF CAVITIES}

\section{ONE-POINT MP}

One-point MP was a major SRF cavity performance limitation in the 70s. It occurs in regions where RF magnetic field is nearly uniform and RF electric field has a non-zero normal component. MP electrons come back to the emission location after an integer ( $\mathrm{N}, \mathrm{N}$ being the order of MP) multiples of RF period. Fig. 1 illustrates trajectories of MP electrons in a muffin-tin cavity [11].

The MP field levels are determined by the magnetic field and scale like $B_{N} \sim f / N$, where $\mathrm{f}$ is RF frequency. The electron impact energy is determined by the normal component of the electric field $E_{n}$ and has a frequency dependence of $B_{N} \sim E_{n}^{2} / f^{2}$. The synchronism between MP electrons and RF field is stable only for a narrow phase range near zero-cross of the electric field, as a result, MP bands are well separated and each has a narrow width.

Interim olutions to suppress one-point MP include sharpening the corner for pill-box cavities and grooving the surface of MP susceptible regions for muffin-tin cavities. 


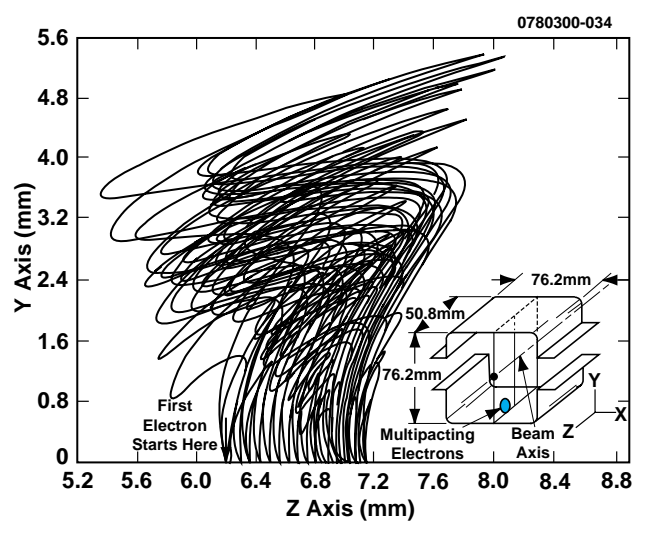

Figure 1: One-point MP in a muffin-tin cavity.

These solutions were both experimentally verified.

The most successful solution to one-point MP is to round the cavity wall to make a spherical cavity [12]. In this geometry, electrons are bent to the equator where energy gain is small. The same MP suppression effect is achieved in the elliptical cavity shape yet with added mechanical strength [13]. The spherical/elliptical cavity shapes have become the mainstream for $\beta=1$ cavities.

\section{TWO-POINT MP}

Two-point MP [14] occurs in small regions near the equator of a spherical/elliptical cavity. Two points symmetric about the equator are involved. The flight time between impacts is an odd-integer ( $2 \mathrm{~N}-1, \mathrm{~N}$ being the order of MP) multiples of half period. Fig. 2 shows the trajectory of twopoint MP electrons in a $1.3 \mathrm{GHz}$ TESLA cavity [17]. The synchronism is maintained by the magnetic field and MP levels scale linearly with frequency $B_{N} \sim f /(2 N-1)$. The impact energy for the $1^{\text {st }}$ order two-point MP is in the range of $30-50 \mathrm{eV}$, irrespective of RF frequency. The synchronism between MP electrons and RF field is stable for a wide range $\left(20^{\circ}-40^{\circ}\right)$ of phase angle and hence the MP barriers are wider compared with that of one-point MP.
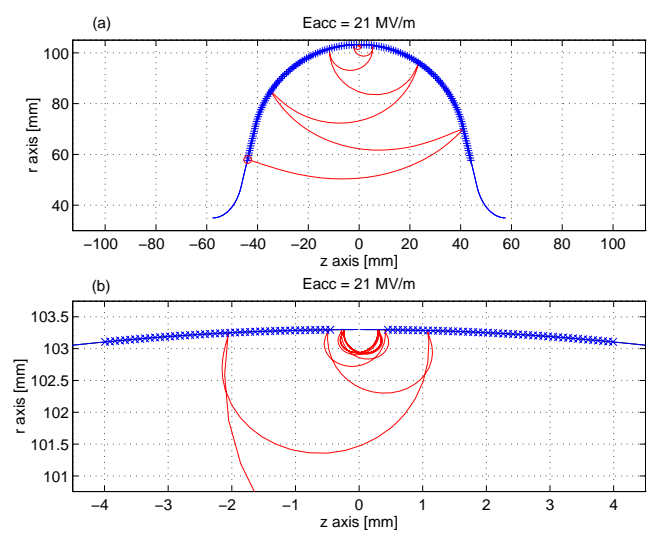

Figure 2: Two-point MP in a single-cell $1.3 \mathrm{GHz}$ TESLA cavity. Note resonant trajectories in (b).

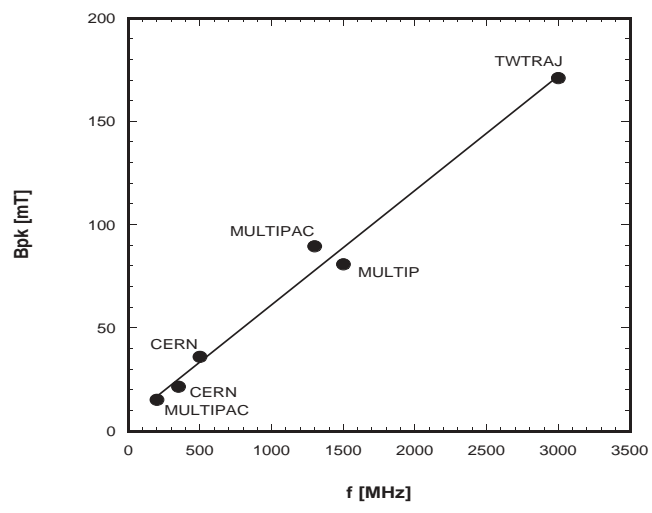

Figure 3: Calculated peak magnetic fields of two-point MP ( $1^{\text {st }}$ order) at various frequencies: $200 \mathrm{MHz}$ [18], $350 \mathrm{MHz}$ and $500 \mathrm{MHz}$ [14], $1300 \mathrm{MHz}$ [17], $1500 \mathrm{MHz}$ [19], 3000 $\mathrm{MHz}$ [20]. Labels indicate names of the simulation code.

Two-point MP has been recognized in other $\beta=1$ elliptical cavities for a wide frequency range from $200 \mathrm{MHz}$ to $3000 \mathrm{GHz}$ through numerical simulations supported by experimental evidence [18] [14] [15] [19] [20]. Fig. 3 summarizes the peak magnetic field levels of the $1^{\text {st }}$ order two-point MP at various frequencies. From Fig. 3, the following scaling law can be established for the $1^{\text {st }}$ order two-point MP in spherical/elliptical $\beta=1$ cavities: $B[m T]=5+55 f[G H z]$.

Although two-point MP's are ubiquitous, they are not expected to limit the cavity performance owing to the fact that the impact energy is very close to the first cross-over of the secondary emission yield (SEY) curve, which shifts up after the surface is "cleaned up" by processing.

\section{MP SIMULATIONS FOR COUPLER WAVEGUIDES}

Gradient performance of SRF cavities has been steadily increasing. SRF cavities now demands higher and higher RF power to be delivered by couplers. Since the 90 s, MP in RF couplers emerges to be an issue that needs to dealt with seriously.

\section{COAX LINES}

Simulation studies of MP in coax lines were attempted as early as 1968 [21] with a neglected RF magnetic field. Results presented below are mainly due to the recent work in [22] [23] and particularly in [24] [25]. The dominant MP mode in a coax line is one-sided MP on the surface of the outer conductor as illustrated in Fig. 4. For a narrow power range, two-sided MP across the inner and outer conductor also exists. In SW mode, MP sites are fixed near the maxima of the electric field. In TW mode, MP electrons travel along with the wave. The MP power levels obey the following scaling law: $P \sim(f d)^{4} Z$ (one-sided MP); $P \sim(f d)^{4} Z^{2}$ (two-sided MP), where $f$ is frequency, $d$ the radius of the outer conductor and $Z$ the line impedance. 


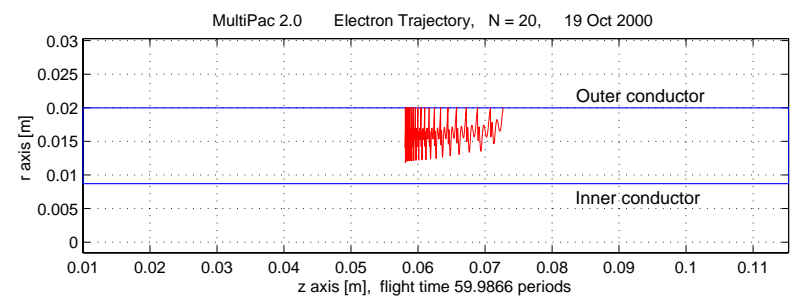

Figure 4: A one-sided MP ( $3^{\text {rd }}$ order) in a $1.3 \mathrm{GHz} 50 \Omega$ coax line with a standing wave.

The impact energy for one-sided MP has a nearly linear dependence on the forward power and can reach $2000 \mathrm{eV}$ for typical cases.

MP in coax lines can be suppressed by simply enlarging the gap between the inner and outer conductor so that a same MP barrier is shifted to a higher power level. Or it can be suppressed by the so called "DC biasing method" [22] [25], which calls for imposing a DC bias voltage on the inner conductor. Full MP suppression can be realized with either polarity of the bias voltage. Full suppression voltage scales like $\left|V_{\text {bias }}\right| \sim f d Z$ and has a typical value of $3 \mathrm{kV}$ for most modern applications. At an intermediate negative bias voltage, the one-sided MP on the surface of the outer conductor can be intensified. This effect is sometimes used to process RF couplers before their operations [26]. It should be pointed out that at an intermediate positive bias voltage, one-sided MP on the inner conductor surface may occur.

\section{RECTANGULAR WAVEGUIDES}

The dominant MP mode in a rectangular waveguide is two-sided MP between broad walls in the high electric field region [27]. MP electrons traverse the gap in an odd-integer $(2 \mathrm{~N}-1, \mathrm{~N}$ being the order of MP) multiples of RF period. Most MP barriers encountered in modern rectangular coupler waveguides are higher order MP's. Fig. 5 illustrates the trajectory of MP electrons in the mid-plane of a 500 $\mathrm{MHz}$ rectangular waveguide with a partially reflected wave. It is shown that MP electrons travel along with the forward wave. The impact energy is linearly dependent on the forward power and can reach $1000 \mathrm{eV}$ for the typical case. Not surprisingly, there is some similarity between rectangular waveguide MP and parallel plate MP. It is justified that MP power levels obey the following scaling law: $P \sim(f b)^{4}$, where $f$ is RF frequency and $b$ the narrow dimension of the waveguide.

MP in rectangular waveguide can be suppressed by opening cut-off slots on broad walls and can be suppressed by the "DC magnetic biasing" method [28]. The magnetic biasing method asks for imposing a weak longitudinal magnetic field ( $\sim 20$ Guass). Full suppression can be realized with either polarity of the bias field. The full suppression bias field for the TW mode scales like $B_{\text {bias }} \sim \sqrt{P /\left(f a b^{3}\right)}$, where $P$ is the forward power, $a$ the

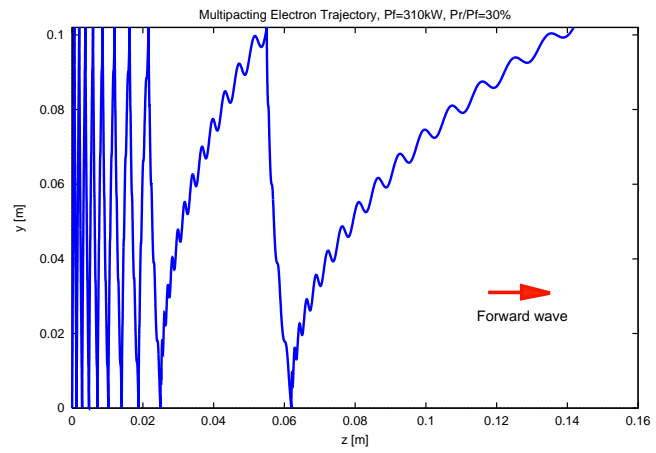

Figure 5: Two-sided MP ( $5^{\text {th }}$ order) in a $500 \mathrm{MHz}$ rectangular waveguide with a partially reflected wave. The trajectory starts from the origin.

wide dimension and $b$ the narrow dimension of the waveguide [29]. MP in rectangular waveguide can be intensified at an intermediate bias field due to oblique incidence. This effect may be used to process waveguides.

\section{CERAMIC WINDOWS}

A full coverage of MP on a ceramic is out of the scope of this paper. However, this subject should not be neglected. Simulation studies show that classical resonant MP occurs on the single ceramic surface or between the ceramic surface and surrounding metal surfaces [30] [31]. Recent theoretical and numerical work by Kishek and Lau [32] point out that due to charge effect, MP occurs on a ceramic surface in a non-resonant fashion and hence for a much wider power range. Coating with a low SEY material is a remedy.

\section{ALGORITHMS FOR MP SIMULATIONS}

The algorithms of MP simulations are illustrated in Fig. 6. For a given geometry, a virtual electron is launched at a known field level from a known surface location with

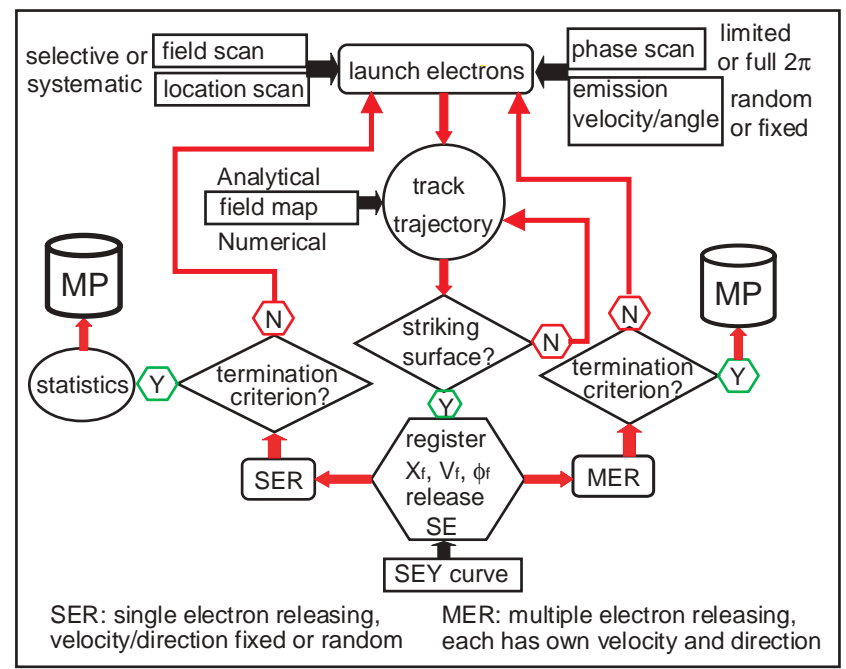

Figure 6: MP algorithm. 
respect to a chosen phase of the RF field. The kinetic energy and direction of the electron at emission are also specified. The trajectory of the virtual electron is tracked by solving its equations of motion, which are coupled ODE's. Numerical integration are made by various methods such as leap-frog or Runge-Kutta. RF fields in the structure are obtained analytically or numerically (imported from an external field solver or supplied by the MP code itself). ${ }^{1}$ At each integration step, judgment is made to check if the electron strikes a surface (this is the major difference from regular ray tracing). If the answer is no, the integration goes on to the next step. If the answer is yes, the impact location, the velocity of the electron and the phase angle of the RF field upon impact are registered. Then the virtual electron is re-emitted from the impact site and its tracking is continued until its next impact with a surface, and so on. After a certain number (usually 30 - 50) of impacts have been made, the tracking is stopped ${ }^{2}$ and the following calculation is performed,

$$
e_{N}=\prod_{i}^{N} \delta_{i}
$$

where $N$ is the total number of impacts, $i$ is the index for each impact, and $\delta_{i}$ is the SEY, calculated for each impact according to the corresponding kinetic energy and direction of the electron upon impact. $e_{N}$ is nothing but the important concept of enhanced counter function, introduced in [23]. For a virtual electron that can make $\mathrm{N}$ times of impact, $e_{N}>0$.

The above described process is repeated for a large set of combined launching parameters: RF field, launching location, phase angle, launching energy and launching angle. After all the launching possibilities are exhausted, MP susceptible zone is constructed. For each field level, $e_{N}$ 's of all virtual electrons that can make $\mathrm{N}$ impacts are summarized and then normalized to the total number of launched electrons for this field level. MP is predicted for the field levels at which $e_{N}>1$ (the physical significance of this criterion is apparent).

Once a MP field level is determined, the MP location(s) can also be pin-pointed by analyzing the impact locations that have been registered. With the MP field level and location both determined, calculations are repeated to trace out the trajectory of MP electrons and to find out the time of flight between impacts (the order of MP).

MP simulations in the past are performed with selective field levels and locations for which MP is already accurately observed by experiments. Today, MP simulations need to deal with untested RF structures, for which MP susceptible field levels and locations are not known. A

\footnotetext{
${ }^{1}$ For apparent reasons, it is essential to have high quality numerical fields near surfaces.

${ }^{2}$ Tracking of a virtual electron can be terminated before $\mathrm{N}$ times of impacts are made for various reasons. For example, if the virtual electron is re-emitted in a retarding field, it may be pulled back to the surface shortly after the emission and hit the surface with a rather low (a few eV) energy. In this case, tracking of this virtual electron should be aborted and another virtual electron is launched. For the aborted electron, $e_{N}=0$.
}

systematic scan of field levels and locations is required for launching virtual electrons.

\section{SER AND MER}

Most of today's MP codes adopt the single electron releasing (SER) scheme in modeling secondary electron emission. This means that there is only one virtual electron before and after impact (in fact, there is only ONE virtual electron at any instant). Upon re-emission, a fixed kinetic energy (typically $2-5 \mathrm{eV}$ ) and a fixed direction (usually normal to the surface) are assigned to the virtual electron. Assignment of these quantities are sometimes done in a random fashion according to the velocity and angular distribution function of secondary emission.

Multiple electron releasing (MER) scheme was adopted by Ben-Zvi et al. [9] in their MP studies of a pill-box SRF cavity in 1973. In this case, when a virtual electron impacts a surface with sufficient energy, multiple virtual electrons (number determined by SEY) are re-emitted. Each new virtual electron has its own initial energy and direction assigned by a Monte Carlo process which considers the velocity and angular distribution function of secondary emission. Ultimately, there is a large number of virtual electrons being tracked simultaneously. MP is predicted when a threshold number of electrons is reached.

\section{EXTERNAL AND INTERNAL PARAMETERS}

Scanned launching parameters can be divided into two groups: external and internal. External parameters include RF field, RF frequency, and dimensions of the RF structure (which determine launching locations). Internal parameters include phase angle, emission energy, and emission direction. MP codes differ mainly in the way of scanning internal parameters. Some codes limit the phase angle to $[\pi, 2 \pi]$, which means launching is allowed only in an accelerating field. In fact, electrons emitted in a retarding field can also escape from the surface, because of the finite initial energy $(2-5 \mathrm{eV})$. The importance of this effect on the bandwidth of MP barriers is explicitly examined theoretically in [6] and numerically in [28]. It is emphasized here that allowing launching, and re-emission, of virtual electrons for the full $2 \pi$ phase angle range is essential. ${ }^{3}$

\section{CODES AND VERIFICATIONS}

There exist several codes (2D and 3D) for MP simulation (see Krawczyk [16]). When analytical RF fields are not available, an EM field solver is needed. Some codes use external field solvers such as MAFIA, OSCAR2D, SUPERFISH and SuperLANS and some use integrated internal solvers. Although most codes are not distributed, some codes are available for interested users [33] [34].

\footnotetext{
${ }^{3}$ Of course the electron will be pulled back to the surface if it experiences an extended period of deceleration. Such an electron will be aborted.
} 
Verifications of code predictions have been brought about by recent experiments. There exists good agreement for two-point MP in elliptical $\beta=1$ SRF cavities [17] [18] and low $\beta$ cavities [35] when a pessimistic SEY is used. Simulation results on an array of low $\beta$ cavities are consistent with test results [36]. However, some experimentally observed MP barriers can not be predicted, even by using an exaggerating SEY [35] [18].

Predictions on MP in coupler waveguides agree qualitatively with experiments. A better agreement is evident for a fresh waveguide than for a "processed" waveguide. [37] [38]. Measured MP bands are usually continuous, in contrast to well separated bands predicted by simulations. During recent measurements of RF couplers for SNS, only 2 harmless MP barriers were observed, despite 7 barriers being predicted by simulations [39].

\section{RECOMMENDATIONS}

Major reasons causing discrepancies between simulation predictions and measurement results are listed below,

- Lack of SEY data of actual surfaces (at RT and low temperatures) for impact energy range of $20-60 \mathrm{eV}$.

- Truncated internal parameters. For example: forbidden emission in a retarding field.

- Negligence of angular and velocity distribution of secondary emssion.

- Negligence of MP dispersing effect such as space charge effect.

Corresponding recommendations for improving MP simulations are listed below,

- Need help from surface scientists to provide realistic SEY data for impact energy range of $20-60 \mathrm{eV}$.

- Allow re-emission for $2 \pi$ phase angle.

- Implement the angular and velocity distribution of secondary emission through a Monte Carlo process. An MER algorithm is a good candidate.

- Include space charge effect - may eventually ask for PIC simulations.

- Develop advanced 3D codes for MP simulations of complicated RF stuctures, like low $\beta$ resonators.

\section{CONCLUSION}

Numerical simulations have played a critical role in understanding and suppression of MP in SRF cavities and RF coupler waveguides. MP simulation algorithms have advanced in the past decade and a comprehensive MP survey of RF structures is now possible. Simulation predictions are well supported by experiments in most cases. Reasons for the remaining discrepancies are identified. It is necessary to implement internal parameters like angular and velocity distributions of secondary emission and to implement space charge effect. This might be best achieved by using an MER algorithm.

\section{REFERENCES}

[1] P.T. Farnsworth, J. Franklin Institute, 218, 411 (1934).

[2] E.W.B. Gill and A. von Engel, Proc. R. Soc. London, A192, 446 (1948).

[3] A.J. Hatch and H.B. Williams, JAP, 25, 417 (1954); PR, 112, 681 (1958).

[4] K. Krebs and H. Meerbach, Ann. Physik, 15, 189 (1955).

[5] H. Tamagawa, E.T.J. of Japan, September 1957, 93 (1957).

[6] V.D. Shemelin, Sov. Phys. Tech. Phys., 31, 1029 (1986).

[7] J.R.M. Vaughan, IEEE Trans. Electron Devices, 35, 1172 (1988).

[8] MP is primarily perceived as a deleterious effect in the SRF community, despite its useful application in other areas.

[9] I. Ben-Zvi, J.F. Crawford, J.P. Turneaure, IEEE Trans. Nucl. Sci., NS-20, 54 (1973).

[10] C.M. Lyneis, H.A. Schwettman, J.P. Turneaure, APL., 31, 541 (1977).

[11] H. Padamsee, A. Joshi, JAP, 50, 1112 (1979).

[12] U. Klein, D. Proch, Proc. of the Conference on Future Possibilities for Electron Accelerators, N1-17 (1979).

[13] P. Kneisel et al., NIM, 188, 669 (1981).

[14] W. Weingarten, Proc. of the 2nd SRF Workshop, 551 (1984).

[15] E. Somersalo, P. Ylä-Oijala, D. Proch, J. Sarvas, Particle Accelerators, 59, 107 (1998).

[16] F.L. Krawczyk, Proc. of the 10th SRF Workshop, (2001).

[17] R. Ylä-Oijala, Particle Accelerators, 63, 105 (1999).

[18] R.L. Geng et al., these Proceedings, Paper TPAB049.

[19] J. Knobloch, W. Hartung, H. Padamsee, Proc. of the 8th SRF Workshop, 1017 (1997).

[20] P. Fabbricatore et al., Proc. of the 7th SRF Workshop, 385 (1995).

[21] J.P. Budlinger, A. Laisne, NIM, 61, 253 (1968).

[22] J. Tückmantel et al., PAC1995, p.1642.

[23] E. Somersalo, P. Ylä-Oijala, D. Proch, PAC1995, P. 1500.

[24] P. Ylä-Oijala, TESLA Report, TESLA 97-20, (1997).

[25] P. Ylä-Oijala, TESLA Report, TESLA 97-21, (1997).

[26] Y. Kijima et al., EPAC2000, p.2040.

[27] R.L. Geng, H. Padamsee, PAC1999, p.429.

[28] R.L. Geng, H. Padamsee, V. Shemelin, PAC2001, p.1228.

[29] R.L. Geng et. al, accepted for publication in NIM-A.

[30] S. Yamaguchi, Y. Saito, S. Anami, S. Michizono, IEEE Trans. Nucl. Sci., 39, 278 (1992).

[31] H. Matsumoto, PAC1999, p.536.

[32] R.A. Kishek and Y.Y. Lau, PRL, 80, 193 (1998).

[33] P. Ylä-Oijala, D. Proch, Proc. of the 10th SRF Workshop, (2001).

[34] http://www.fieldp.com/

[35] G. Devanz, EPAC2000, p.1366.

[36] W. Hartung et al., Proc. of the 10th SRF Workshop, (2001).

[37] G. Devanz, PRST-AB, 4, 012001 (2001).

[38] R.L. Geng et al., EPAC2002, p.2238.

[39] M. Stirbet, High Power Coupler Workshop, Jlab, (2002). 